\title{
Eruption of a Quiescent Filament on Feb. 18, 2003
}

\author{
Xingming Bao and Hongqi Zhang \\ National Astronomical Observatories, Chinese Academy of Sciences, \\ Beijing 100012, China
}

An eruptive process of a quiescent filament on Feb. 18, 2003 that initiated a CME was recorded by the full disk $\mathrm{H} \alpha$ telescope in HSOS (Huairou Solar Observing Station) and by the Solar and Heliospheric Observatory (SOHO). The eruption revealed a typical process of energy release and dense plasma ejection caused by magnetic instability. Filament eruptions, flares and CMEs are believed to be the same magnetic eruption and reconnection event in three different stages. This feature was shown more clearly during eruption of the quiescent filament because of the simple and regular magnetic line-of-sight field in photosphere compared to active regions with complex magnetic configurations. The filament was located near the solar northwest limb (N 30-40, W 40-80) on Feb. 18,2003 with height about $11000 \mathrm{~km}$. It began to erupt at 02:00 UT. Bright ribbons appeared at the magnetic feet of the filament in EIT/195 at 02:30 UT. The post-eruption loops formed at 04:00 UT and lasted about 6 hours. The CME appeared on EIT/C2 images at 02:30UT with an $\Omega$ shape, just the same as the filament erupted. The speed of mass ejected of the filament was about $250 \mathrm{~km} / \mathrm{s}$. We also notice that the height of the filament increased and the magnetic fields with opposite polarity moved towards each other before the filament erupted. The filament eruption reveals that the filament may be held in the corona by the twisted magnetic fields which also keep the cool plasma from escaping the solar surface. If the twisted field was broken by some magnetic instabilities, the filament would erupt. The presence of post-eruption loops implies a transition process from non-potential (twisted) to potential field magnetic configuration in the filament. 